\section{THU0483 BONE MINERAL DENSITY AND PREVALENCE OF OSTEOPOROSIS IN HIV-INFECTED PATIENTS IN COMPARISON WITH A REFERENCE SPANISH POPULATION: THE IMPORTANCE OF LOCAL NORMATIVE RANGES}

C. Sobrino $^{1}$, J. Loarce ${ }^{1}$, F. López ${ }^{1}$, J. Gallego ${ }^{2}$, M.J. Vivancos ${ }^{2}$, M. Pérez Elías ${ }^{2}$, J. L. Casado ${ }^{2}$, M. Vazquez ${ }^{1}{ }^{1}$ Rheumatology; ${ }^{2}$ Infectious Diseases, Hospital Universitario Ramón y Cajal, Madrid, Spain

Background: HIV-infected patients have less bone mineral density (BMD) than non-HIV population. According to current guidelines, the normal BMD reference range is that derived from the US NHANES III (National Health and Nutrition Examination Survey) and from BMDCS-Hologic. However, local variations of BMD could change the interpretation of bone loss and the prevalence of osteoporosis $(\mathrm{OP})$ in this population.

Objectives: To compare the BMD in HIVinfected Spanish patients with values from healthy Spanish population and to estimate the prevalence of OP in this patients, based on the local data.

Methods: This was a cross-sectional study in a homogenous cohort of HIVinfected patients (RyC cohort, EC 009/17). Data of femoral neck and spine BMD were obtained by DXA (Dual-Energy X-ray Absorptiometry) from 949 patients (241 women, $25 \%$ ) and compared with the results of a nationally representative Spanish cohort (14 centres) including 2442 subjects (1305 women, 53\%) aged 20-80 years.

Results: Overall, mean age was 45.4 years (women, 46.5 years). The Spanish reference cohort showed a reduced BMD $(-7 \%, 3 \%-12 \%)$ in comparison with NHANES, especially in middle aged women. HIVinfected patients had a lower BMD than Spanish cohort in both spine and femoral neck localizations for the different age strata $(p<0.001)$, not significant in femoral neck for males aged 30-39 $(n=152$ patients, $0.848 \mathrm{vs} 0.869 \mathrm{gr} / \mathrm{cm} 2 ; \mathrm{p}=0.07)$. Using NHANES data, the prevalence of OP was $26 \%$ in women in the Spanish cohort and $41 \%$ in our HIV population. By contrast, if the Spanish data are used as normative range, the prevalence of OP decreased in HIV females, significant between $40-60$ years (from $26.5 \%$ to $23.5 \%$ in spine, $p<0.01$ ). Although OP in spine was less frequent in males by using Spanish data ( $p<0.01$, OP decrease from $17.8 \%$ to $6.5 \%$ ), there was more OP in femoral neck in males older than 40 years (from $13.5 \%$ to $21 \%$ in $60-69 ; p<0.01$ ). Conclusions: Our data demonstrate that HIV-infected patients had a significantly reduced BMD in comparison with both US and Spanish reference data for all the age strata. However, the prevalence of osteoporosis could change if local representative cohorts are used as normative data, with almost $30 \%$ of patients being reclassified.

Disclosure of Interest: None declared

DOI: 10.1136/annrheumdis-2018-eular.7096

\section{THU0484 REBOUND-ASSOCIATED MULTIPLE VERTEBRAL FRACTURES AFTER DISCONTINUATION OF DENOSUMAB: NINE CASES REPORT}

E. Fernández, D. Benavent, G. Bonilla, I. Monjo, S. García, M. Bernad, A. Balsa, P. Aguado. Rheumatology, Hospital Universitario La Paz, Madrid, Spain

Background: Denosumab (DNB) is an IgG monoclonal antibody with high affinity for RANKL that transiently decreases bone resorption. It increases bone mineral density (BMD) and decreases turnover markers, reducing fracture risk in patients with osteoporosis (OP). Several cases of rapid loss of BMD, severe bone turnover rebound (BTR) and associated fractures have been reported after DNB discontinuation. A clinical alert has been generated from these findings.

Objectives: To evaluate the clinical and demographic features in a case series of patients with multiple vertebral fractures after discontinuation of denosumab.

Methods: An observational descriptive study analysing data from nine patients with multiple vertebral fractures after DNB discontinuation that were admitted to our Rheumatology service between 2015 and 2017.

Results: Nine women with postmenopausal OP that received treatment with DNB were included (table 1). Mean age at treatment onset was 66.9 \pm 8.0 SD years. Four of them had previous fragility fractures, and one of them had risk factors for secondary OP (treatment with aromatase inhibitors, case 8). Eight patients $(88.9 \%)$ had already received treatment other than DNB. Mean DNB received doses was $6 \pm 1.6$ SD. Mean T-score before treatment was $-2.6 \pm 0.6$ SD for femoral neck (FN) and $-3.0 \pm 1.3 \mathrm{SD}$ for lumbar spine. Mean T-score $\pm \mathrm{SD}$ after 6 months or more of DNB discontinuation was $-3.5 \pm 0.8$ and $-3.3 \pm 1.8$, respectively. Bone turnover markers (BTM) remained elevated (CTX mean 0.143 $\pm 0.663 \mathrm{SD} \mathrm{ng} / \mathrm{ml}$ and P1NP $152.1 \pm 122.4 \mathrm{SD} \mathrm{ng} / \mathrm{ml}$ ). There were a total of $47 \mathrm{frac}-$ tures, and all occurred spontaneously. Most affected vertebrae were D9, D12, L3 $y$ L5. Two patients underwent vertebroplasty, suffering both of them vertebral fractures after surgery.

Table 1 Clinical features of the patients

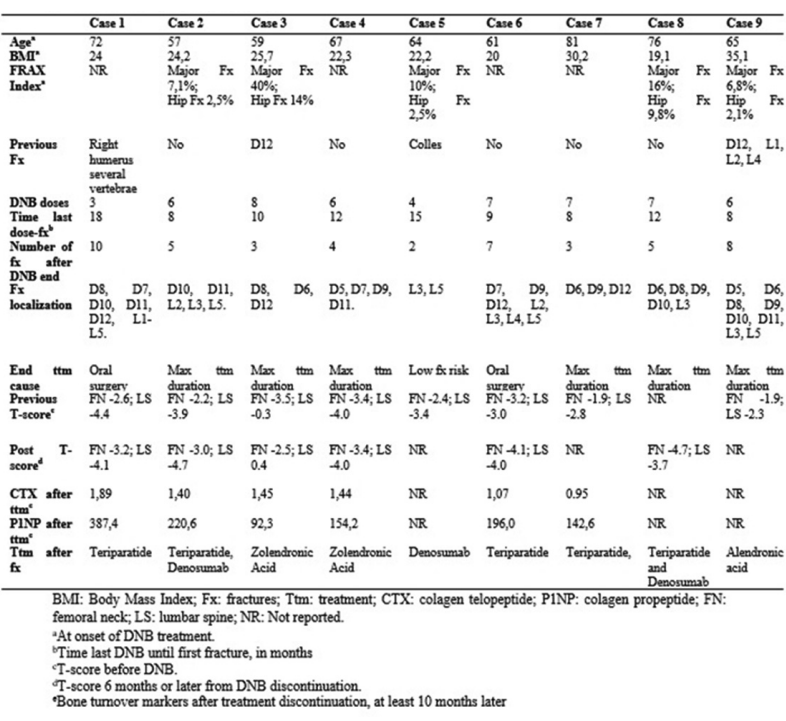

Conclusions: This report of nine cases suffering multiple vertebral fractures early after the interruption of the DNB highlights the emerging concern on the subject in the scientific community and the need to clarify its pathogenic mechanism ("rebound effect") and support in solid evidence the new recommendations on its management.

Disclosure of Interest: None declared

DOI: 10.1136/annrheumdis-2018-eular.7046

\section{THU0485 PREDICTING 1-YEAR MORTALITY AFTER A FRAGILITY HIP FRACTURE - THE EXPERIENCE OF A FRACTURE LIAISON SERVICE}

F. Aguiar ${ }^{1,2}$, M. Guerra ${ }^{3}$, S. Ganhão $0^{1,2}$, D. Gonçalves ${ }^{4}$, T. Martins-Rocha ${ }^{1,2}$, R. M. Ferreira ${ }^{1,2}$, A. Águeda ${ }^{2,5}$, R. Vieira ${ }^{3}$, G. Terroso ${ }^{1}$, C. Vaz $^{1,2}$, L. Costa $^{1}$.

${ }^{1}$ Rheumatology, Centro Hospitalar São João, Porto; ${ }^{2}$ Faculty of Medicine of Porto University, Oporto; ${ }^{3}$ Rheumatology, Centro Hospitalar Gaia/Espinho, Gaia; ${ }^{4}$ Rheumatology, Centro Hospitalar Trás-os-Montes e Alto Douro, Vila Real; ${ }^{5}$ Rheumatology, Centro Hospitalar Baixo Vouga, Aveiro, Portugal

Background: Hip fractures are the most serious outcome of osteoporosis and are a leading public health concern due to the associated increase in morbidity and mortality, loss of independence and financial burden.

Objectives: The aim of this study was to investigate possible predictive factors of 1 year mortality in patients with fragility proximal femur fracture referred to a fracture liaison service (FLS).

Methods: Patients aged $>65$ years admitted in Orthopaedics department with a fragility hip fracture were evaluated and referred to the Rheumatology outpatient clinic. In this setting patients were evaluated with laboratorial and imagiological workup and afterwards anti-osteoporotic therapy was started. We retrospectively collected information regarding patients hospitalised from March 2015 to March 2017 and conducted univariate and multivariate analysis to determine possible predictive factors of 1 year mortality.

Results: 522 patients were included, with a median age of 84 years (range 65104), $416(79.7 \%)$ females. The median time to intervention was 2 days (range 0 44) and median hospital stay 10 days (range $0-175$ ). Median Charlson Comorbidity Index (CCl) score was 5 (range 2-13). In-hospital mortality was $6.3 \%$ and overall 1 year mortality was $16.7 \%$.

In the univariate analysis, the factors significantly associated with death in one year time were male gender, $\mathrm{CCl}$ score $>5$, previous physical limitation in daily activities, walking disability previously and 2 months after the fracture, longer time to surgery ( $>48$ hour), conservative treatment and previous osteoporosis diagnosis. In multivariate analysis previous physical limitation in daily activities (OR 2.1 Cl 1.22-3.62; $p=0.007$ ) and walking disability 2 months after the fracture (OR 4.23 , $\mathrm{Cl} 1.73-10.37 ; \mathrm{p}=0.002$ ) were independent predictors of 1 year mortality. Conclusions: In this study, in-hospital mortality was similiar to what has been described in literature but 1 year mortality was slightly lower. Previous physical limitation and walking disability 2 months after the fracture were independent risk factors for 1 year mortality. These findings should be confirmed in larger, prospective studies with a control group.

Disclosure of Interest: None declared

DOI: 10.1136/annrheumdis-2018-eular.6813 